\title{
UN CAS DE BRONCHOSPASME INDUIT PAR LA SUCCINYLCHOLINE
}

\author{
Natalis Bele-Binda, M.D. et Franco Valeri, M.D. ${ }^{*}$
}

Mrle. P. Nz., 18 aNs, dossier n 36468 est admise aux Cliniques Universitaires Lovanium le 1 mars 1966 pour mal de Pott dorso-lombaire D-11, D-12-L-1.

(1) Familiaux: on ne note rien de particulier.

(2) Personnels: Malaria.

L'examen sanguin pratiqué chez elle le 21 mars avait montré l'existence d'une éosinophilie à 24 pour cent et des microfilaires. Le 15 avril 1966 on décide de faire un curetage plus greffe dorse-lombaire sous anesthésie générale.

Détails de l'anesthésie: Prémédication: atropine $0,5 \mathrm{mg}$. Pethidine $50 \mathrm{mg}$.

Induction: thiopentone $200 \mathrm{mg}$ succinylcholine $100 \mathrm{mg}$. Après injection de succinylcholine, la patiente déclenche un bronchospasme qui nous oblige, après ventilation à $1^{\prime} 0_{2}$ pure, de donner $50 \mathrm{mg}$ de succinylcholine supplémentaire pour l'intuber. L'intubation fut laborieuse. Grâce à l'administration de $250 \mathrm{mg}$ d'aminophylline $\mathrm{Iv}$ et de $4 \mathrm{mg}$ de dexamethasone, le bronchospasme et levé 30 minutes plus tard mais l'on décide de remettre l'intervention.

Le 19 avril la patiente revient en salle d'opération pour sa cure de mal de Pott.

La prémedication est faite de nouveau avec de l'atropine $0,5 \mathrm{mg}$, pethidine 50 $\mathrm{mg}$, mais avec cette fois-ci un suppositoire d'aminophylline.

L'induction s'effectue au Thalamonal (Innovar) éliminant ainsi le thiopentone.

A l'injection de $100 \mathrm{mg}$ de succinylcholine, la patiente entre de nouveau en bronchospasme; on réussit de vraincre ce bronchospasme avec de l'aminophylline $250 \mathrm{mg}$ et du dexamethasone $4 \mathrm{mg} \mathrm{w}$.

\section{COMMENTAires}

La survenue d'un bronchospasme à deux reprises chez une même patiente après injection de succinylcholine nous a poussé à penser que cette drogue était la cause sinon le facteur déclenchant de ce bronchospasme.

Notre hypothèse s'est renforcée lorsque l'enquête menée pour découvrir des antécédents asthmatiques chez la patiente et dans la famille s'était révélée négative. L'éosinophylie 24 pour cent constatée lors de la numération globulaire s'explique par la présence des microfilaires dans le sang.

Les myorésolutifs: d.tubocurarine, gallamine triothiodide, succinylcholine (Suxamethonium) libérent à des degrés différents de l'histamine. Le Suxamenthonium (Succinylcholine) libère 1 à 2 pour cent de la quantité d'histamine libérée par la $\mathrm{d}$. tubocurarine qui est la plus histaminogène des trois drogues. ${ }^{2}$ C'est l'histamine libérée qui agit sur les effecteurs localisés dans les muscles bronchiques qui provoque la complication bronchique.

${ }^{\star}$ Cliniques Universitaires Lovanium, Kinshasa, République Démocratique du Congo. 
Expérimentalement chez le chien, ${ }^{1}$ la succinylcholine est capable d'induire un bronchospasme mais en clinique les bronchospasme déclenchés par succinylcholine sont très rares. ${ }^{2-6}$ Le premier cas de bronchospasme par succinylcholine a été rapporté par Fellini et Col. en 1963. ${ }^{6}$

\section{RÉSUMÉ}

Un cas de bronchospasme induit par la succinylcholine chez une patiente de 18 ans sans antécédents asthmatiques est rapporté.

Après une revue de littérature les auteurs concluent que de tels cas sont rares en clinique. 